\title{
Uso de Mapa de Características na Avaliação de Textos Curtos nos Ambientes Virtuais de Aprendizagem
}

\author{
Marcos A. Spalenza ${ }^{1}$, Matheus A. Nogueira ${ }^{1}$, Márcia G. de Oliveira ${ }^{2}$, Elias de Oliveira ${ }^{1}$ \\ ${ }^{1}$ Programa de Pós-Graduação em Informática - Universidade Federal do \\ Espírito Santo (UFES) - 29.075-910 - Vitória - ES - Brasil \\ marcos.spalenza@gmail.com, elias@lcad.inf.ufes.br
}

${ }^{2}$ Centro de Referência em Formação e em Educação a Distância (CEFOR) - Instituto Federal do Espírito Santo (IFES) - 29.040-860 - Vitória - ES - Brasil

\begin{abstract}
This paper proposed a feedback model for Virtual Learning Environments - VLE built automatically after the teacher's evaluation. This feedback results from the similarities among texts of the same grade and shows markings on more informative words for each student's submissions. The system, indeed, encourages the discussion of discipline content and enables all course participants learning collaboratively. Providing this dynamic correction, the teacher coordinates a discussion of results and suits teaching-learning methods.
\end{abstract}

Resumo. Neste artigo é proposto um modelo de feedback para Ambientes Virtuais de Aprendizagem - AVA construído automaticamente após a avaliação do professor. Este feedback resulta da similaridade dos textos de mesma nota e são apresentadas aos estudantes marcações nas palavras mais informativas em cada uma das submissões. O sistema, na verdade, estimula a discussão dos conteúdos da disciplina e capacita todos os participantes do curso a verificarem o aprendizado de forma colaborativa. Ao proporcionar essa dinâmica de correção o professor coordena a discussão dos resultados e adequa os métodos de ensino-aprendizagem.

\section{Introdução}

Durante as atividades cotidianas, as pessoas em geral, produzem uma enorme quantidade de dados que podem ser trabalhados para a redução da carga de trabalho e a construção de conhecimentos de grande valor. A produção de informações por sistemas computacionais permite a todos interessados mais facilidade na tomada de decisões e a redução do esforço em trabalhos específicos. Com o avanço tecnológico, observa-se um aumento constante no poder de processamento, o que torna mais viável obter informações em tempo real.

Dentre as atividades aonde é viável implantar este processamento de dados, incluise a rotina escolar. Por conta disso, é possível, por exemplo, auxiliar os professores apresentando feedbacks individuais aos participantes da disciplina com seu critério de avaliação nas atividades. O processamento para produção de feedbacks, se apoiado por sistemas inteligentes, agilizaria a apresentação dos resultados e liberaria o professor para mais avaliações formativas. Desta forma, cada aluno recebe antecipadamente os resultados individuais, enquanto uma breve discussão esclareceria as possíveis dúvidas ainda 
V Congresso Brasileiro de Informática na Educação (CBIE 2016)

Anais do XXVII Simpósio Brasileiro de Informática na Educação (SBIE 2016)

restantes. Assim, com uma análise colaborativa da atividade entre os alunos, permitese que o professor se encarregue de identificar precisamente as necessidades de reforços durante o processo de ensino e de aprendizagem.

Segundo [Fluminhan et al. 2013], "o feedback é um importante recurso de comunicação, porém tem sido negligenciado por professores que, por falta de tempo em sala de aula, por excesso de trabalho, por desconhecimento de sua eficácia ou por inabilidade em tratá-lo como um ato de orientação, de acompanhamento e de avaliação, simplesmente se omitem em praticá-lo de forma regular e consistente". Portanto, ofeedback é um instrumento educacional de grande valor para que o aluno adquira sensibilidade aos fatores diretamente ligados à melhora de desempenho. Para isso o professor deve trabalhar para apresentar instrumentos alternativos de reforço e discussões em torno do conteúdo a cada parte da tarefa. Muito embora seja algo que depende de muito tempo de dedicação do docente, os comentários do tutor são fatores que contribuem para o desenvolvimento do estudo.

Com o objetivo de criar feedbacks automaticamente, o sistema proposto neste artigo, baseia-se nas submissões dos alunos e na respectiva nota atribuída pelo professor. Este sistema, portanto, cria um mapa de características que seleciona termos relevantes nos textos dentre os grupos de nota apresentados pela avaliação do professor. No feedback enviado aos alunos, então, estará o texto do aluno em cores destacando os termos que representam cada nota. Estes destaques permitem, ao observar as submissões, interpretar trechos específicos que caracterizam a avaliação do professor no conjunto das respostas dos alunos, identificando os conceitos estudados na disciplina.

Em cada submissão marcada, o estudante pode comparar com seus colegas a equivalência das respostas e discutir o resultado da avaliação do professor. O docente, por consequência, abstrai a resposta dos usuários ao ver como foi o padrão de avaliação identificado, reavaliando seu método segundo a correspondência das submissões dos estudantes. Dessa forma, o professor caracteriza seu processo de avaliação endossando a justiça do seu método avaliativo. É possível ainda que o professor, ao visualizar todas as atividades, liste quais conteúdos não tiveram sua devida atenção e devem, portanto, ser reforçados.

É importante que cada discente conheça seu modelo de aprendizado para elaborar rotinas próprias de estudo em aula e pós aula. Isto ocorre com maior frequência no Ensino a Distância $(\mathrm{EaD})$, onde o contato pessoal com o professor é reduzido. Segundo [Bischoff 2000], "o feedback constante e consistente nas sala de aula on-line pode estimular o engajamento ativo através de técnicas tais como questionar pressupostos, discordar de alguns pontos, e destacar pontos bem analisados". Portanto, é neste modelo de ensino que o estudante depende mais das instruções do professor para assimilar qual é seu nível de compreensão do conteúdo.

Este trabalho está organizado conforme a seguinte ordem. Na Seção 2, temos os trabalhos relacionados à proposta. Na Seção 3, descrevemos o mapa de características como ferramenta. Na Seção 4, discutimos os resultados da aplicação do sistema. Na Seção 5, concluímos com as considerações finais e os trabalhos futuros.

\section{Trabalhos Relacionados}

O feedback é uma ação do professor que avalia o conhecimento do aluno qualitativamente. Este processo é muito estudado pela pedagogia, dada a importância do aluno compreender 
V Congresso Brasileiro de Informática na Educação (CBIE 2016)

Anais do XXVII Simpósio Brasileiro de Informática na Educação (SBIE 2016)

o conteúdo e do professor em relatar sua metodologia de observação avaliativa.

Foi através do cognitivismo, que o feedback se destacou. Conforme [Fluminhan et al. 2013], neste período "o erro deixou de ser simplesmente descartado e passou a ser uma fonte de referência sobre os processos cognitivos do aluno, e desta forma, tornou-se parte integrante do processo de ensino e aprendizagem". Este é um instrumento que contribui para que o ambiente de ensino seja mais dinâmico independente do modelo pedagógico adotado. Para [?], "o feedback é fundamental para o EaD e a notoriedade do ensino on-line, onde o diálogo entre professor e aluno deve superar o isolamento de modo a contribuir com o processo de ensino/aprendizagem".

Além do tempo de correção, a criação de comentários individuais geralmente demanda muito tempo de análise do professor. A sobrecarga de trabalho, principalmente relacionada ao excesso de atividades para avaliação, reflete diretamente na redução gradativa do número de exercícios aplicados na disciplina [Oliveira et al. 2015].

A criação de feedbacks automáticos é evidenciada pela avaliação automática de códigos de programação. Com o desenvolvimento destes sistemas, os comentários de correção foram fundamentais para complementar a informação passada aos alunos. Sistemas como os descritos por [Alves and Jaques 2014] e [Oliveira et al. 2015] geram relatórios individuais conforme a execução dos códigos dos alunos. Enquanto [Raabe et al. 2015] estuda a avaliação qualitativa dos feedbacks gerados automaticamente por este tipo de sistema. Neste estudo ainda é apresentado o impacto positivo deste modelo de correção no processo de aprendizagem dos discentes.

$\mathrm{Na}$ análise de textos, a compreensão é fundamental para gerar comentários diretos sobre o conteúdo. O estudo de respostas discursivas feito por [Leffa 2003] descreve a dificuldade de compreender a sintaxe, a ordem e o significado textual para corresponder adequadamente às submissões dos alunos. A proposta deste artigo, diferentemente das abordagens de extração linguística ou comentários genéricos, faz uso das avaliações do professor para marcar trechos que expliquem aos participantes o padrão de avaliação.

\section{Mapa de Características}

O mapa de características foi uma técnica aqui desenvolvida com base no método de avaliação de respostas discursivas. Com esta ferramenta, o professor corrige cada questão com notas que serão interpretadas como classes no sistema para, automaticamente, identificar trechos relevantes para cada uma delas.

Para apresentar informações valiosas sobre as tarefas, a nossa ferramenta busca primeiramente a resposta mínima de cada classe. Sabendo que o professor já efetuou a correção, questões com notas equivalentes são agrupadas. Em cada grupo é extraído quais trechos estão presentes em grande parte das submissões com a nota em questão.

Para a coleta de dados utilizamos o Ambiente Virtual de Aprendizagem - AVA Moodle ${ }^{1}$. O uso deste tipo de plataforma é muito conhecido dos cursos EaD e conta também com aplicações de sucesso em turmas presenciais. Com isso, todos os usuários destes ambientes são o público-alvo da ferramenta proposta neste artigo, independente do modelo pedagógico de ensino. A submissão de tarefas em texto on-line no AVA permite

\footnotetext{
${ }^{1}$ Modular Object-Oriented Dynamic Learning Environment (Moodle) - http: / / moodle. org
} 
V Congresso Brasileiro de Informática na Educação (CBIE 2016)

Anais do XXVII Simpósio Brasileiro de Informática na Educação (SBIE 2016)

que a atividade, quando finalizada, seja coletada pelo plug-in descrito por [Pissinati 2014] para ser processado pelo sistema.

Após cada coleta de dados, as submissão dos estudantes são pré-processadas para padronização dos textos de entrada. Cada grupo, formado pela nota da avaliação do professor, é enviado ao Algoritmo Genético para seleção de características e, consequentemente, a redução de dimensionalidade. Com o resultado deste processo é gerado o mapa de características como relatório individual, com a identificação dos trechos considerados relevantes em cada grupo. As palavras selecionadas recebem cores conforme a nota dos textos ao qual foi destaque sendo esta cor atribuída à nota de forma aleatória. Estas cores identificam a nota através das palavras na visualização em um documento HTML da submissão. O HTML gerado é enviado diretamente ao campo de feedback do Moodle aonde pode ser vista no resumo da atividade, pelo professor e pelo aluno. O procedimento realizado pelo sistema é apresentado na Figura 1.

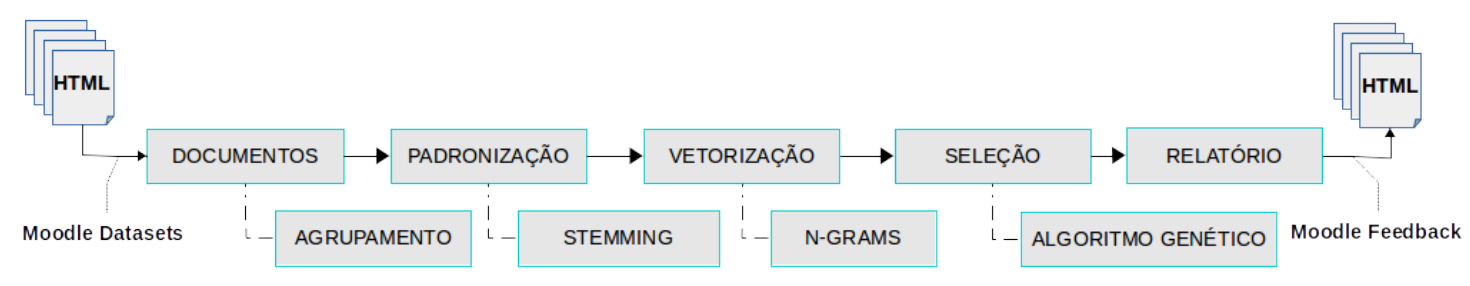

Figura 1. Estrutura de processamento do sistema com dados do Moodle.

\subsection{Identificação do Conjunto Resposta}

Para capturar o padrão das atividades, o aplicativo normaliza todas as submissões dos alunos. Um pré-processamento utiliza os procedimentos de stemming, remoção de stopwords e a eliminação de sinais gráficos. Isso garante que todas as ocorrências das palavras remetam à uma mesma forma quando verificada sua frequência.

Para análise computacional de cada submissão dos usuários, cada documento $d$ é interpretado como um vetor de termos. Um documento $d$ em cada grupo $D$ $\left(d_{1}, d_{2}, d_{3}, \ldots, d_{i}\right)$ é representado pela frequência de suas palavras, em uma base de dados de $i$ documentos. Para cada documento $d$ é computada a ocorrência de conjuntos de ter$\operatorname{mos} t\left(t_{1}, t_{2}, t_{3}, \ldots, t_{j}\right)$ onde foram encontrados $j$ termos distintos nos documentos. Dada essa ocorrência individual, os conjuntos são formados pela sequência de cada um destes termos. O sistema foi configurado para testar de 2 até 5-grams objetivando identificar sequências relevantes na estrutura de resposta dos alunos. Neste processo os $n$-grams garantem que palavras individuais repetidas sejam consideradas com um peso proporcional. Desta forma, cada resposta dos estudantes é processada como uma estrutura numérica multidimensional capaz de ser comparada com os demais documentos.

Após a contagem dos n-grams o sistema tenta otimizar uma função de densidade de cada agrupamento. Individualmente, os vetores de documentos por classe de nota são enviados a um Algoritmo Genético para redução de dimensionalidade. Nesta metaheurística, cada identificador de uma sequência de termos é selecionada por um vetor binário. Conforme [Baeza-Yates and Ribeiro-Neto 2011] a métrica mais usual para medir a distância entre dois documentos e utilizada nestes experimentos foi a similaridade de cosseno. Este cálculo é dado pela Equação 1. 


$$
\operatorname{sim}\left(d_{1}, d_{2}\right)=\frac{d_{1} \cdot d_{2}}{\left\|d_{1}|||| d_{2}\right\|}
$$

Então, o valor complementar à média do cálculo de similaridade entre todos os documentos indica a homogeneidade do conjunto de mesma nota. Então, o fitness medido a cada geração do Algoritmo Genético é dado pela média de dissimilaridade interna do grupo. O objetivo desta otimização, portanto, é minimizar as diferenças dos documentos visando encontrar a possível estrutura de resposta representativa.

Após a definição de todos os melhores $n$-grams para cada nota, o sistema estabelece um peso específico para cada termo $t$. Com resultado do Algoritmo Genético é considerada a frequência do termo $t$ na classe para atribuir um peso por palavra, a qual indica a relevância deste termo para a nota. $\mathrm{O}$ peso atribuído às palavras, posteriormente, serão utilizados como a graduação da cor destas mesmas palavras no relatório em HTML.

\subsection{Aplicações}

Dentre as aplicações desse método de feedback podemos destacar questões aonde o enunciado exige como respostas listas de fatos, respostas semi-objetivas, respostas abertas mas com alvo único, etc. Isso se deve pela tendência a todos os textos dos alunos a respostas similares, mesmo que existam divergências internas em cada classe. Assim, a avaliação do professor é validada pela existência de padrões possíveis de serem observados.

Nas questões subjetivas, de opinião e de múltiplas respostas podem ocorrer que várias discussões distintas sobre um dado assunto sejam tratadas como coerentes mesmo que os textos não sejam similares. A subjetividade do critério de correção também é um problema adicional, a validade do discurso depende da interpretação do leitor. Assim, todo fator que independe da coesão e equivalência textual foge da abordagem da ferramenta.

Apesar de alguns limites na identificação textual, resultados do sistema de construção do mapa de características serão discutidos na Seção 4.

\section{Resultados}

Para exemplificar o uso da ferramenta proposta neste trabalho utilizamos diferentes questões aplicadas nos cursos da Universidade Federal do Espírito Santo (UFES). Cada base de dados foi enviada pela plataforma Moodle para o sistema identificar as respostas e os resultados são discutidos à seguir.

Em cada base de dados, o professor corrigiu com notas discretas, interpretadas posteriormente como grupos. A Tabela 1 apresenta em cada atividade o número de submissões e de grupos identificados pelo professor.

Os códigos de cada uma destas atividades representam cursos diferentes da UFES. $M T P C$ é um código referente à disciplina de Métodos e Técnicas de Pesquisa Científica. $\mathrm{O}$ código TECII é utilizado para a sala da disciplina de Tecnologia da Informação II, aonde basicamente é ensinada a construção de páginas WEB para os alunos de Arquivologia. Já FIL refere-se ao curso de Filosofia e Ética também lecionada nesta universidade.

Para verificar a situação de cada uma destas bases de dados antes e depois da execução do algoritmo foram medidas a similaridade interna de cada etapa. A seguir, na 
V Congresso Brasileiro de Informática na Educação (CBIE 2016)

Anais do XXVII Simpósio Brasileiro de Informática na Educação (SBIE 2016)

\begin{tabular}{l|cc}
\hline Base de Dados & Submissões & Grupos \\
\hline MTPC-13-220 & 15 & 4 \\
TECII-5-8 & 46 & 3 \\
TECII-5-129 & 22 & 3 \\
TECII-5-177 & 27 & 1 \\
FIL-13-220 & 31 & 5 \\
\hline \hline
\end{tabular}

Tabela 1. Bases de dados utilizadas para teste do algoritmo

Tabela 2 observamos a classe, número de submissões e a similaridade interna anterior e posterior de cada questão.

\begin{tabular}{r|c|c|c}
\hline Classe & \multirow{2}{*}{ Submissões } & \multicolumn{2}{c}{ Similaridade. Interna } \\
\cline { 3 - 4 } & & Anterior & Posterior \\
\hline \multicolumn{4}{|c}{ MTPC-13-220 } \\
\hline 100.00 & 3 & 0.667 & 0.889 \\
90.00 & 10 & 0.777 & 0.684 \\
80.00 & 1 & 1.000 & 1.000 \\
50.00 & 1 & 1.000 & 1.000 \\
\hline
\end{tabular}

\begin{tabular}{r|c|c|c}
\multicolumn{5}{c}{ TECII-5-8 } \\
\hline 100.00 & 29 & 0.807 & 0.901 \\
90.00 & 11 & 0.768 & 0.688 \\
0.00 & 6 & 0.709 & 0.972 \\
\hline
\end{tabular}

\begin{tabular}{r|c|c|c}
\multicolumn{4}{|c}{ TECII-5-129 } \\
\hline 100.00 & 18 & 0.933 & 0.987 \\
80.00 & 1 & 1.000 & 1.000 \\
20.00 & 3 & 0.652 & 0.735 \\
\hline
\end{tabular}

\begin{tabular}{l|l|l|l}
\multicolumn{4}{|c}{ TECII-5-177 } \\
\hline 100.00 & 27 & 0.655 & 0.982 \\
\hline
\end{tabular}

\begin{tabular}{r|c|c|c}
\multicolumn{5}{c}{ FIL-13-220 } \\
\hline 100.00 & 17 & 0.913 & 0.859 \\
90.00 & 4 & 0.749 & 0.871 \\
80.00 & 5 & 0.800 & 0.960 \\
60.00 & 2 & 0.500 & 0.750 \\
40.00 & 3 & 0.667 & 0.889 \\
\hline \hline
\end{tabular}

Tabela 2. Diferença de similaridade interna em cada grupo antes e depois da aplicação do Algoritmo Genético.

A Tabela 2 apresenta a diferença de similaridade antes e depois do processamento 
V Congresso Brasileiro de Informática na Educação (CBIE 2016)

Anais do XXVII Simpósio Brasileiro de Informática na Educação (SBIE 2016)

do algoritmo nas bases de dados. Das 16 classes dispostas entre estas atividades, 10 tiveram êxito na redução de dimensionalidade enquanto em 3 ocorreu diminuição da similaridade. A redução da similaridade representa que um trecho do texto foi identificado em grande parte das submissões, indicando a seleção de uma resposta mínima. Enquanto, o aumento sugere que a redução textual não atinge todos os textos do grupo e algum ruído pode afetar o agrupamento. Tal ruído pode ser resultado da aplicação dos critérios discutidos na Seção 3.2. Nestas bases de dados também foram encontradas classes com submissões únicas. Tais classes não podem ser otimizadas pelo Algoritmo Genético pois, nestes grupos, o algoritmo não pode realizar comparações de similaridade. Assim, neste caso especificamente, todos os termos identificados após o pré-processamento referenciam à classe.

Utilizaremos como exemplos mais específicos as questões TECII-5-177, TECII5-8 e FIL-13-220 para apresentar as situações de aplicação deste sistema.

A base de dados TECII-5-177 foi utilizada como um teste inicial do algoritmo. Com o enunciado "Segundo o autor, quais são os tradicionais profissionais da informação?" e baseando-se em [Arellano and Andrade 2006] todos os alunos responderam corretamente a questão. Apesar disto, a similaridade interna anterior ao processamento da base de dados era de 0,655 e foi ampliada para 0,982. Uma amostra das submissões podem ser vistas na Tabela 3 , com o identificador do aluno, a nota representante e a resposta submetida.

\begin{tabular}{|c|c|c|}
\hline ID & Nota & Resposta \\
\hline $289-4033$ & 100.0 & $\begin{array}{l}\text { Segundo o autor os arquivistas e biblio- } \\
\text { tecários considerados os tradicionais profissio- } \\
\text { nais da informação }\end{array}$ \\
\hline $260-4028$ & 100.0 & $\begin{array}{l}\text { Conforme o texto Os profissionais da } \\
\text { informação são Arquivistas e Bibliotecários }\end{array}$ \\
\hline $258-4027$ & 100.0 & $\begin{array}{l}\text { Os arquivistas e bibliotecários considerados } \\
\text { os tradicionais profissionais da informação }\end{array}$ \\
\hline $186-4011$ & 100.0 & Os arquivistas e bibliotecários \\
\hline $184-4009$ & 100.0 & Arquivistas e Bibliotecários \\
\hline $176-4002$ & 100.0 & Arquivista e Bibliotecário \\
\hline $173-4000$ & 100.0 & Arquivistas e Bibliotecários \\
\hline $169-3996$ & 100.0 & $\begin{array}{l}\text { Segundo os autores são os arquivistas e bibli- } \\
\text { otecários }\end{array}$ \\
\hline
\end{tabular}

Tabela 3. Identificação inicial de trechos relevantes do sistema exemplificada com a amostra da base de dados TECII-5-177.

Nesta atividade o professor avaliou como correta todas as respostas que citavam arquivistas e bibliotecários. Assim, esta base de dados foi muito importante para o desenvolvimento do algoritmo pela uniformidade das respostas e a consistência dos trechos.

Após o teste, um enunciado interessante para aplicação foi "Quais são os elementos base no modelo proposto pelo DCC?", da atividade TECII-5-8. Nesta questão os alunos responderam com base no artigo [Sayão and Sales 2012]. O resultado gerou uma 
V Congresso Brasileiro de Informática na Educação (CBIE 2016)

Anais do XXVII Simpósio Brasileiro de Informática na Educação (SBIE 2016)

base de dados com muitas submissões maiores que exemplo anterior. Abaixo, nas Figuras 2, 3 e 4 vemos as submissões de menor similaridade dentro de cada agrupamento.

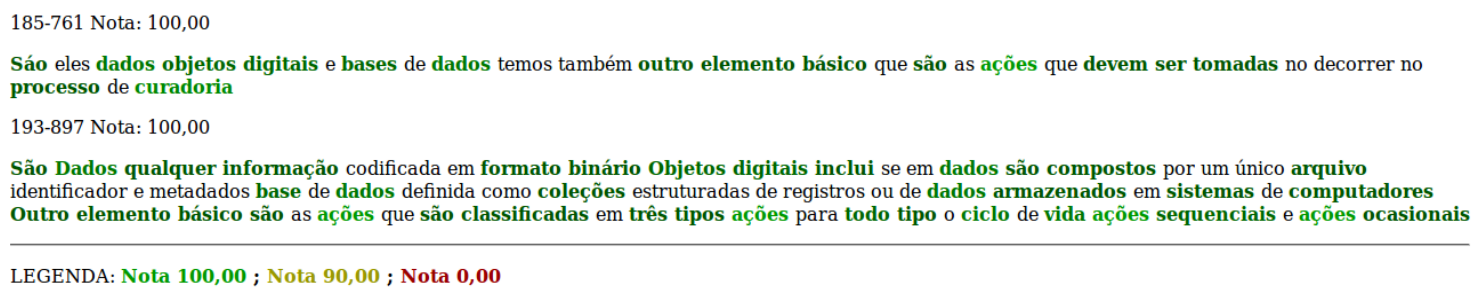

Figura 2. Respostas mais dissimilares da nota 100,00 para a atividade TECII-5-8.

24-85 90,00

Os elementos chaves do modelo são dado objetos digitais e base de dados

$32-8690,00$

Os elementos base que ocorre no modelo proposto pelo DCC são objetos digitas e base de dados

LEGENDA: Nota 100,00; Nota 90,00; Nota 0,00

Figura 3. Respostas mais dissimilares da nota 90,00 para a atividade TECII-5-8.

176-849 0,00

Os elemento básico do modelo são as ações que devem ser tomadas no decorrer do processo de curadoria O modelo do DCC classifica as ações em três tipos Ações para todo o ciclo de vida Açốes sequenciais e Ações ocasionais

28-78 0,00

O modelo proposto pelo DCC está orientado para o planejamento das atividades de curadoria nas organizações ou consórcios ajudando a garantir que todos os passos do ciclo serão cumpridos

LEGENDA: Nota 100,00; Nota 90,00; Nota 0,00

Figura 4. Respostas mais dissimilares da nota 0,00 para a atividade TECII-5-8.

Ao comparar as Figuras 2, 3 e 4, podemos compreender a avaliação da atividade. Além de cores específicas por grupo de nota, identificamos entre as palavras tons mais claros que representam maior destaque e tendem a ser mais relevantes. Nesta atividade, a palavra ações garantia a diferença entre as notas 100,00 e 90,00. Por estar presente em todos os envios de nota 100,00, esta palavra foi selecionada como relevante e recebeu uma tonalidade mais clara de verde. Nestas figuras, vemos que a nota 90,00 não teve conteúdo próprio. Isso se deve ao fato de todas as suas palavras relevantes representam uma nota majoritária, no caso, a nota 100,00. Enquanto isso, os documentos avaliados com 0,00 contém trechos equivalentes às outras notas, mas pela avaliação do professor, concluímos que eles não eram essenciais.

Para esta atividade, a resposta estará correta quando os estudantes citarem em suas submissões dados, objetos digitais, bases de dados e ações. Todas estas palavras foram identificadas com a nota 100,00. Para diferenciar as notas 100,00 e 90,00, o professor atribuía a maior nota às questões que citavam ações. Enquanto isso, a ausência de dados, objetos digitais e bases de dados recebia a nota 0,00 do professor.

Apesar de identificar o critério do professor pelas marcações, vamos observar a amostra da atividade FIL-13-220 apresentada na Figura 5. Nesta imagem, vemos as submissões de menor similaridade antes e depois do processamento da questão. Os alunos 
V Congresso Brasileiro de Informática na Educação (CBIE 2016)

Anais do XXVII Simpósio Brasileiro de Informática na Educação (SBIE 2016)

\author{
3-5752 100,00 \\ Todas as filosofias do período helenístico apesar das diferenças de método desejam proporcionar ao \\ homem a paz de espírito do grego ataraxia Este interesse comum está ligado ao fato de que no helenismo \\ perde se as referencias da cidade estado construída pela participação política como aconteceu na \\ democracia ateniense Resta a filosofia orientar como viver bem nesse novo mundo \\ $374-6110100,00$ \\ A busca pela paz de espírito através da ética e sabedoria \\ 379-5928 100,00
}

No Helenismo houve perda da importância política individual por isso as principais escolas filosóficas da época procuravam determinar diretrizes racionais fugindo do sofrimento em busca da felicidade e do bem estar

LEGENDA: NOTA 100,00; NOTA 90,00; NOTA 80,00 ; NOTA 60,00

Figura 5. Respostas de menor similaridade, antes e depois do processamento, para 0 grupo da nota 100,00 da atividade FIL-13-220.

respondiam conforme o conteúdo estudado na disciplina a seguinte pergunta: "A expansão do Império de Alexandre, o Grande, aumentou as fronteiras da cultura grega. Começa assim o período denominado helenismo em que surgem diversas escolas filosóficas como o cinismo, o epicurismo e o estoicismo. Há entre essas escolas uma característica em comum. Diga que característica é essa.”.

Dentre os submissões, a resposta de ID 3-5752 foi enviada pelo professor. Podemos observar como o sistema correspondeu às expectativas e a direcionou à nota 100,00, interpretando somente os documentos enviados e sua respectiva avaliação. Enquanto isso, os alunos ID 374-6110 e ID 379-5628 tem a mesma nota mas enviaram textos completamente diferentes. Conforme a Tabela 2, a queda de similaridade interna (de 0,913 para 0.859) pode explicar o que ocorre neste conjunto de submissões.

Para analisar esta redução, a Figura 5 apresenta as relações de menor similaridade do agrupamento 100,00 antes e depois da otimização do sistema. Enquanto uma é realmente a resposta ideal, pois foi entregue pelo professor (especialista), as outras duas são completamente distintas. Durante a correção, o professor visava encontrar alguma referência à paz de espírito pelos estudantes. Como este texto não foi encontrado na tarefa do aluno ID 379-5628, vemos que mais critérios foram adotados. Nestes casos, a resposta de cada um é validada pela correção colaborativa. Durante a análise dos estudantes, as dúvidas recorrentes devem ser discutidas em sala para efetivar o aprendizado.

Nesta tarefa, as considerações do tutor, por não terem referência diretamente textuais, tornam o método de avaliação um pouco mais subjetivo. Dentre essas considerações, podemos citar a forma de escrita e o domínio do conteúdo apresentado. Desta forma, o resumo textual entregue pelo algoritmo não foi, neste último exemplo, tão eficaz quanto às anteriores, apesar das marcações adequadas.

\title{
5. Conclusão
}

A estratégia proposta neste trabalho seleciona trechos descritivos das atividades para endossar o critério de avaliação do professor. Quando há uma tendência nas respostas dos alunos, a possibilidade de apoio do sistema é elevada. Caso contrário, a subjetividade das respostas ou do critério de correção pode apresentar visões diferentes do conteúdo e, consequentemente, reduz a qualidade por classe de nota no tratamento das submissões. 
V Congresso Brasileiro de Informática na Educação (CBIE 2016)

Anais do XXVII Simpósio Brasileiro de Informática na Educação (SBIE 2016)

Como trabalhos futuros a partir deste, podemos validar este método observando como cada agrupamento foi avaliado. A aplicação tende a resultados positivos quando temos grande similaridade interna. Para isso, além da eliminação do ruído causado pelas respostas incoerentes, será necessária a verificação de sua existência conforme os índices de similaridade. Outra integração para usabilidade é a criação de um relatório descrevendo cada atividade.

Desta forma, é importante salientar que a colaboração do sistema apresenta potencial para ser mais uma ferramenta pedagógica para as dinâmicas de sala de aula. Em sua aplicação, espera-se que os alunos discutam a convergência ou a divergência dos resultados com seu material de apoio. Ao tutor, é possível pelo AVA verificar se o mapa de características gerado foi equivalente às expectativas e aprová-lo. Caso a análise do sistema seja diferente da visão do professor, este pode a qualquer momento gerar manualmente ofeedback individual.

\section{Referências}

Alves, F. P. and Jaques, P. (2014). Um Ambiente Virtual com Feedback Personalizado para Apoio a Disciplinas de Programação. In Simpósio Brasileiro de Informática na Educação (SBIE), volume 25, pages 1078-1082, Dourados, MS, Brasil.

Arellano, M. A. M. and Andrade, R. S. (2006). Preservação Digital e os Profissionais da Informação. $d g z, 7(5)$.

Baeza-Yates, R. and Ribeiro-Neto, B. (2011). Modern Information Retrieval: The Concepts and Technology Behind Search. Addison Wesley.

Bischoff, A. (2000). The Elements of Effective On-line Teaching: Overcoming the Barriers to Success. In White, K. W. and Weight, B. H., editors, The On-line Teaching Guide, pages 57-72. Allyn \& Bacon, Boston, Londres.

Fluminhan, C. S. L., Arana, A. R. A., and Fluminhan, A. (2013). A Imporância do Feedback como Ferramenta Pedagógica na Educação à Distância. Colloquium Humanarum, 10(Especial):721-728.

Leffa, V. J. (2003). Análise Automática da Resposta do Aluno em Ambiente Virtual. Revista Brasileira de Linguística Aplicada, 3:25 - 40.

Oliveira, M. G., Oliveira, E., and Nogueira, M. A. (2015). Sistema de Apoio à Prática Assistida de Programação por Execução em Massa e Análise de Programas. In Workshop sobre Educaçcão em Informática (WEI), volume 23, Recife, PE, Brasil.

Pissinati, E. M. (2014). Uma Proposta de Correção Semi-Automática de Questões Discursivas e de Visualização de Atividades para Apoio à Atuação do Docente.

Raabe, A. L. A., Jesus, E. A., Andrei, H., and Pelz, F. (2015). Avaliação do Feedback Gerado por um Corretor Automático de Algoritmos. In Simpósio Brasileiro de Informática na Educação (SBIE), volume 26, pages 358-366, Maceió, AL, Brasil.

Sayão, L. F. and Sales, L. F. (2012). Curadoria Digital: Um Novo Patamar para a Preservação de Dados Digitais de Pesquisa. infoSoc, 22(3):179 - 191. 